interventional trials. Participants in this CF study had a field-based estimate of exercise capacity and objective measurement of physical activity at each study visit.

Methods Participants wore a pedometer for 7 days prior to each visit. At each visit, London patients performed a standard incremental shuttle-walk test and Edinburgh patients a modified shuttle test (in which running was allowed). Data are expressed as mean (SD). Results Data were analysed from 192 patients over 648 visits. Age at enrolment was 24 (11.9) years (London) and 20.8 (9.9) (Edinburgh) ( $\mathrm{p}=0.052) ; \mathrm{FEV}_{1}$ was 67 (17.7)\% and 79 (19.5)\% for each site respectively $(p<0.001)$. Daily step count at visit 1 was 7491 (2887) in London and 8872 (4089) in Edinburgh $(p=0.04)$ and this difference persisted across subsequent visits. The coefficient of variation (CV) in step counts between visits was $21.3 \%$. Number of shuttles completed in London was 61 (15), and Edinburgh 90 (33) with no trend over the four visits (CV=10 and $16 \%$ respectively). In Edinburgh there was a correlation between mean step count and the number of completed shuttles $(r=0.46, p<0.001)$. Step count from both sites, and the number of shuttles completed in Edinburgh, correlated with $\mathrm{FEV}_{1} \%$ predicted $(\mathrm{r}=0.24, \mathrm{p}<0.001$ and $\mathrm{r}=0.27, \mathrm{p}<0.001$ respectively) and with age $(\mathrm{r}=-0.28, \mathrm{p}<0.001$ and $\mathrm{r}=-0.30, \mathrm{p}<0.001$ respectively). Such correlations were either weaker or not observed in London, however, in this group, number of shuttles correlated with height $(\mathrm{r}=0.51$, $\mathrm{p}<0.001)$.

Conclusions No changes were detected in exercise capacity or daily activity levels over time. Between site differences were observed in both measures; however, these populations also differ in age and $\mathrm{FEV}_{1}$. The modified shuttle test performed in Edinburgh appeared to better correlate with clinical markers than the standard incremental shuttle test performed in London, and is independent of height. We believe that testing exercise capacity is important in CF and we plan to investigate the other testing methods in the run up to our Multi Dose Gene Therapy Trial.

Funding Funded by the UK CF Trust.

\section{COPD: sputum and exacerbations P110 OUANTITATIVE PCR-BASED DETECTION AND QUANTIFICATION OF ATYPICAL BACTERIA AT BASELINE AND EXACERBATION OF COPD}

doi:10.1136/thx.2010.150987.11

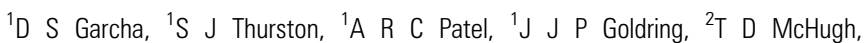
${ }^{1} \mathrm{G}$ C Donaldson, ${ }^{1} \mathrm{~J}$ A Wedzicha. ${ }^{1}$ Academic Unit of Respiratory Medicine, University College London Medical School, London, UK; ${ }^{2}$ Centre for Clinical Microbiology, University College London Medical School, London, UK

Introduction Airway bacterial infections are associated with exacerbations of COPD. The potential role of atypical bacteria as a trigger for exacerbations is not well understood. Atypical bacteria such as Chlamydophila pneumoniae (CP), Legionella pneumophila (LP) and Mycoplasma pneumoniae (MP) are difficult to culture as they are intracellular pathogens. LP can be detected by urinary antigen, and serology can be performed for MP, but these techniques give no indication as to the atypical bacterial load. Quantitative PCR (qPCR) offers an alternative approach to identification and quantification of bacteria in sputum.

Methods Multiplex qPCR was used to detect and quantify CP, LP and MP in 238 samples prospectively collected from 87 patients in the London COPD Cohort: mean $( \pm \mathrm{SD})$ age $71.4( \pm 8.1)$; predicted $\mathrm{FEV}_{1} 43.4 \%$ ( $\left.\pm 17.5 \%\right)$; male gender $47.9 \%$; current smoker $49.2 \%$. Baseline $(n=104)$, exacerbation $(n=95)$, and follow-up $(n=39)$ samples were tested: Baseline was defined as at least 6-weeks without exacerbation, and exacerbation was defined as 2 consecutive days of two symptoms (Anthonisen criteria), at least one of which is a major symptom (dyspnoea; sputum purulence; sputum volume). Follow-up involved taking samples 2 or 5 weeks postexacerbation onset. Using a qPCR developed by our clinical diagnostic service, the CP, MP and LP gene targets were RNA-polymerase $\beta$-chain; P1 adhesin protein; and MIP respectively. Routine microbiological analysis was also performed on these samples.

Results No samples were positive for the atypical organisms using culture. With qPCR analysis 6/238 samples (six separate patients) were positive for LP $(2.5 \%)$, four at baseline and two at exacerbation/follow-up. One baseline sample was positive for MP $(0.42 \%)$, and no samples were positive for CP. Atypical bacteria were present at $0.83 \%$ of exacerbations. Median (IOR) bacterial load was $4.3 \times 10^{4} \mathrm{cfu} \mathrm{ml}^{-1}\left(2.0 \times 10^{4}-8.55 \times 10^{4}\right)$ for LP PCR-positive samples; the MP-positive sample load was $2.64 \times 10^{7} \mathrm{cfu} \mathrm{ml}^{-1}$

Conclusion Quantitative PCR was more sensitive and informative than standard microbiological culture for the detection of atypical bacteria. Atypical bacteria in sputum were detected at very few exacerbations of COPD; moreover, when they were detected by qPCR, the load was low, indicating little or no significance in the aetiology of these events.

\section{P111 FTIR SPECTROSCOPIC PROFILING OF COPD SPUTUM: IDENTIFICATION OF DISTINCT SPECTRAL SIGNATURES AND CORRELATION TO COPD STATUS}

doi:10.1136/thx.2010.150987.12

${ }^{1} \mathrm{~N}$ Patel, ${ }^{1} \mathrm{P}$ Coleborn, ${ }^{1} \mathrm{~A}$ Hampton, ${ }^{1} \mathrm{~V}$ Campbell, ${ }^{1} \mathrm{M}$ Allen, ${ }^{2} \mathrm{~A}$ Gahkani, ${ }^{1} \mathrm{M}$ Spiteri. ${ }^{1}$ Directorate of Respiratory Medicine, Institute of Science \& Technology in Medicine, University Hospital of North Staffordshire, Stoke on Trent, England; ${ }^{2}$ Bruker UK, Coventry, England

COPD remains common and complex with huge costs on the NHS and disruption to patients' daily lives. This presents a challenge for an effective, non-invasive test to enable determination and monitoring of COPD status. Fourier transform infrared (FTIR) spectroscopy for sputum profiling is timely. FTIR identifies and measures chemical bond vibrations within functional groups in complex biological mixtures by producing infrared absorption spectra. Identifying COPD-relevant spectra in sputum could provide sensitive rapid information on status and exacerbations. To address this, we randomly recruited 102 patients independent of severity. Sputum was collected at initial visit and in each of subsequent 2 weeks. Dyspnoea and sputum scores were obtained; FEV 1 , serum CRP and exhaled NO measured; any intervening chest infection or treatment change documented. Patients was stratified by $\mathrm{FEV}_{1} ; 26$ patients had mild COPD ( $\mathrm{FEV}_{1} \geq 80 \%$ ); 41 moderate $\left(\mathrm{FEV}_{1} 49-79 \%\right)$; 35 severe $\left(\mathrm{FEV}_{1} \leq 50 \%\right)$. FTIR was performed using an Alpha-T spectrometer (Bruker UK); transmission mode in 4000 to $900 \mathrm{~cm}^{-1}$ region; $4 \mathrm{~cm}^{-1}$ resolution. All COPD sputa gave reproducible biological IR spectra with distinct signatures in 5 key regions at $3300-3280 \mathrm{~cm}^{-1}$ (assigned as amide A), 3000-2800 $\mathrm{cm}^{-1}, 1660-1600 \mathrm{~cm}^{-1}$ (Amide I), $\quad 1560-1520 \mathrm{~cm}^{-1}$ (Amide II) and $1180-1000 \mathrm{~cm}^{-1}$ (glycoproteins). Multivariate analysis showed significant correlation between spectral profiles and $\mathrm{FEV}_{1}$ and smoking habit. The accuracy of differentiating mild from severe COPD was consistently greater than $70 \%$ (AUC under ROC curve); specifically we observed peak shifts in Amide A towards $3300 \mathrm{~cm}^{-1}$ as COPD worsened $(p<0.001)$. Also $68 / 102$ patients exhibited a clear band around $2060 \mathrm{~cm}^{-1}$; the remaining 34 showed no band in this region. There was a significant association $(p=0.012)$ between peak presence and COPD severity; with mild COPD patients more likely to have a peak, and severe sufferers having $2060 \mathrm{~cm}^{-1}$ signal absence. $35 / 102$ patients had exacerbations during the study. Separate spectral analysis showed significant increase in glycoprotein max peak during COPD exacerbation $(\mathrm{p}<0.03)$. This study has important implications for future near-patient COPD monitoring. FTIR 
sputum profiling enables non-invasive rapid sampling and evaluation, producing spectral signatures that can differentiate COPD status or predict exacerbation presence.

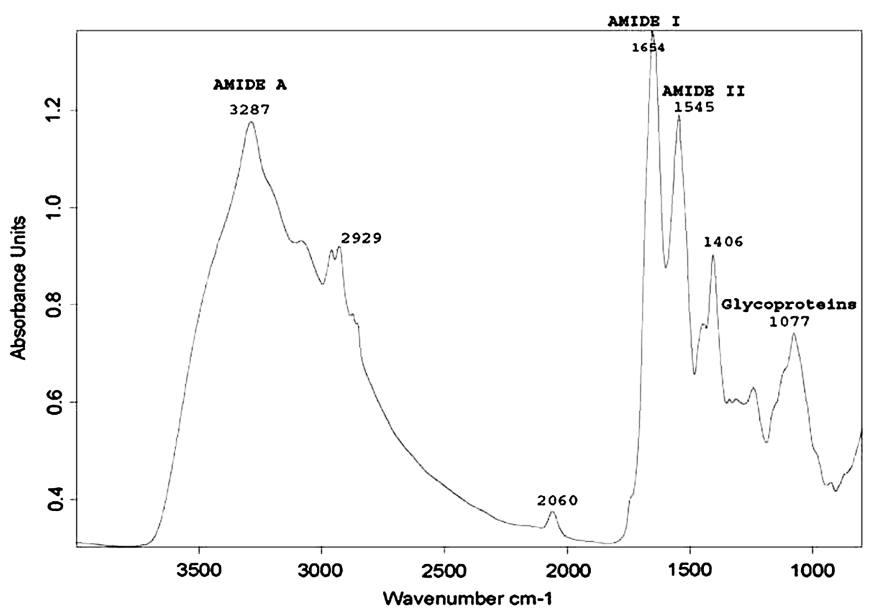

Abstract P111 Figure 1 Example of a typical IR absorption spectrum for COPD sputum.

\section{P112 ASSOCIATION OF SPUTUM PSEUDOMONAS AERUGINOSA (PSA) ISOLATION AND LENGTH OF HOSPITAL STAY IN PATIENTS WITH CHRONIC OBSTRUCTIVE PULMONARY DISEASE (COPD)}

doi:10.1136/thx.2010.150987.13

${ }^{1} \mathrm{~A}$ Ali, ${ }^{2} \mathrm{M}$ Bhattacharya, ${ }^{2} \mathrm{~J}$ Corcoran, ${ }^{3} \mathrm{~B}$ Chakraborty, ${ }^{1} \mathrm{~A}$ Thomas, ${ }^{4} \mathrm{M}$ Raza, ${ }^{1} \mathrm{R}$ Mukherjee. ${ }^{1}$ Department of Respiratory Medicine \& Physiology, Birmingham Heartlands Hospital, Bordesley Green East, Birmingham, UK; ${ }^{2}$ Department of Respiratory Medicine, Milton Keynes Hospital, Milton Keynes, UK; ${ }^{3}$ School of Mathematics, University of Birmingham, Birmingham, UK; ${ }^{4}$ Department of Microbiology, Milton Keynes Hospital, Milton Keynes, UK

Introduction and objectives A lot of effort and investment has been made to improve the length of stay (LOS) in COPD related admissions with variable success. It is known that numbers of hospital admissions and re-admissions have increased. Bacterial infections contribute to acute exacerbation of COPD (AECOPD) in $50 \%$ of cases; PsA is a probable pathogenic organism (PPM) causing acute or chronic infection in severe COPD patients. We set out to examine whether PsA in sputum influenced the length of stay as this PPM is not covered by the routinely used first line antibiotics. Method A retrospective audit was carried out examining the sputum culture results and LOS in all COPD admissions in an acute hospital from 1 January 2009 to 31 December 2009, patients were identified from coding (ICD: J44). Sputum cultures were attempted (and collected in all sputum producers) by a dedicated respiratory team as a routine for all COPD admissions throughout that period, which was established as part of service improvement well before the audit. Results were obtained from Pathology department database. Patients were divided into sputum producing group and nonproducers, Sputum producers were further divided into patients with positive sputum culture of PPM and non-pathogenic organisms. PsA growers were identified. Median LOS was calculated to be 5 days and all groups were compared with this duration as a standard for their LOS.

Results Total admissions with COPD were 332; 203 were sputum producers and 122 had bacterial isolates. There were 37 PsA growers. LOS was seen to be 5 or more days in: 121 sputum producers vs 47 non-producers, $\mathrm{p}=0.000046 .79$ PPM culture positive patients vs 42 without bacterial growth, $\mathrm{p}=0.067$; PsA growers vs. all admissions, $p=0.000744$, PsA vs all sputum producers $p=0.0126$, PsA vs all PPM, $\mathrm{p}=0.041$.

Conclusions PsA infection is associated with significantly longer LOS in hospital. All COPD patients should possibly be screened for PsA infection (and possibly underlying bronchiectasis) to inform the selection of antibiotics, which is likely to reduce LOS and may reduce re-admission rates.

\section{Estimated Distribution of Length of Stay}

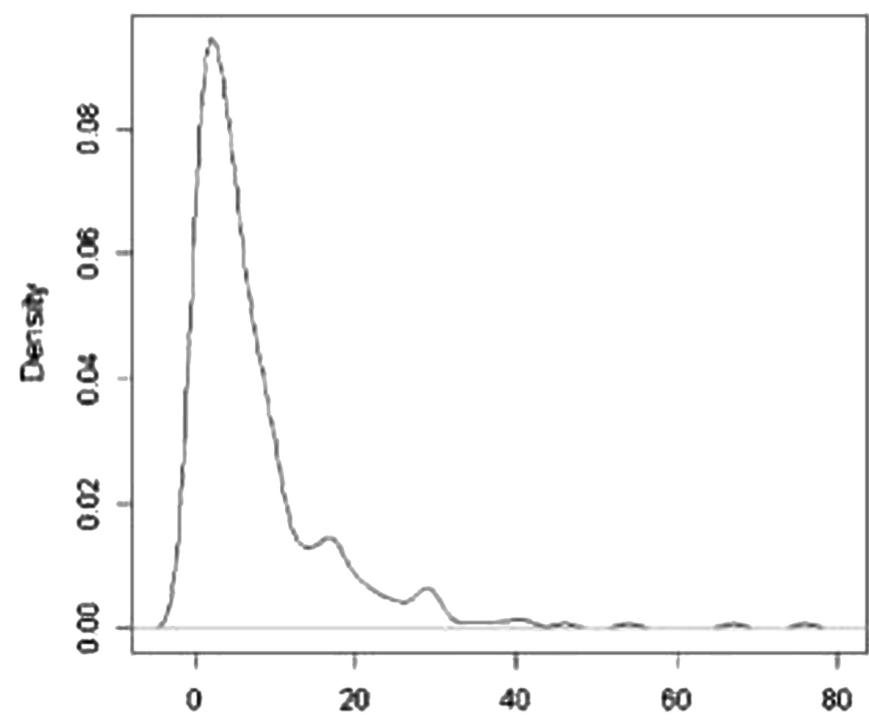

\section{P113 PROCALCITONIN (PCT) IS A SAFE AND RELIABLE BIOMARKER OF BACTERIAL INFECTION IN EXACERBATIONS OF COPD - SO WHY IS IT SO CHALLENGING TO INTRODUCE IT INTO A LARGE UK HOSPITAL?}

doi:10.1136/thx.2010.150987.14

L Burke, M Alhajji, N Todd, J S White. York Hospitals, York, UK

Background Antibiotics have only marginal efficacy in treating acute exacerbations of COPD (AECOPD). There is a large body of evidence that supports the use of PCT as a marker of bacterial infection. In York hospital patients with AECOPD account for a significant proportion of acute admissions and many are treated with antibiotics without strong evidence of a bacterial cause.

Objective To conduct a service evaluation of antibiotic prescribing following the introduction of PCT in patients with AECOPD. To observe attitudes towards and uptake of the test, and identify barriers to implementation.

Methods The evaluation ran from November 2009 to June 2010. Information on PCT was introduced to all physicians, and an algorithm for use was provided. Medical notes were reviewed from patients where PCT was requested. Evidence of documentation of PCT and whether it had been acted upon were recorded along with antibiotic use, length of stay and readmission within 1 month of discharge. Results 54 PCT tests were performed and 49 were included in the final analysis. $32(65 \%)$ of samples were below the cut-off for antibiotics, but were still prescribed in 11 cases. Of those above the threshold for treatment three did not receive antibiotics. PCT was documented and acted upon in only 12 (24\%) patients. Overall, antibiotics were used in $25(51 \%)$ patients. Two patients were readmitted with AECOPD within 1 month of discharge having been managed according to PCT results, with one receiving antibiotics. 24 $(49 \%)$ samples were not reported on the day they were taken.

Conclusion 11 RCTs from different countries enrolling over 3500 patients have demonstrated the feasibility and safety of PCT in 